\title{
Facile Heterogeneously Catalyzed Nitrogen Fixation by MXenes
}

José D. Gouveia, ${ }^{\dagger}$ Ángel Morales-García, ${ }^{\ddagger}$ Francesc Viñes, ${ }^{\dagger}$ José R. B. Gomes ${ }^{*}{ }^{\dagger}$ Francesc Illas, ${ }^{*}$

${ }^{\dagger}$ CICECO - Aveiro Institute of Materials, Department of Chemistry, University of Aveiro, Campus

Universitário de Santiago, Aveiro, Portugal

Departament de Ciència de Materials i Química Física \& Institut de Química Teòrica i Computacional (IQTCUB), Universitat de Barcelona, c/ Martí i Franquès 1-11, 08028 Barcelona, Spain

*Corresponding Authors: jirgomes@ua.pt (JRBG) and francesc.illas@ ub.edu (FI)

\section{Contents}

S1. Additional computational details.

S2. Additional comparison to previous work.

S3. Ammonia synthesis.

S4. Microkinetic simulations.

Table S1. Calculated MXenes lattice parameters.

Table S2. N adsorption energies on the studied MXenes.

Table S3. Energy barrier comparison of the total energy reaction barriers of the $\mathrm{N}_{2}$ dissociation and the hydrogenation reaction between MXenes and (001) Ru surfaces.

Table S4. Energy barriers of the Gibbs free energy reaction profile of ammonia synthesis.

References 


\section{S1. Additional computational details}

The interaction of $\mathrm{N}_{2}$ and subsequent dissociation to adsorbed atomic $\mathrm{N}$ has been considered for a total of 18 MXenes — nine carbides and nine nitrides - with $\mathrm{M}_{2} \mathrm{X}$ stoichiometry, where $\mathrm{M}$ is a transition metal and $\mathrm{X}$ can be carbon or nitrogen. The transition metals examined are in groups IV-VI of the Periodic Table and include Ti, V, Cr, Zr, Nb, Mo, Hf, Ta, and W. The calculated lattice parameters, $a$, of the MXenes are shown in Table S1. The trend is that $a$ decreases along elements of the same period of the Periodic Table and is smaller for nitrides than for carbides. The calculated lattice parameters in Table S1 are in very good agreement with the corresponding values found in the literature. ${ }^{1,2}$

To avoid interaction between replicas of the MXene surface, due to the use of periodic boundary conditions, the total height of the cell, in the direction perpendicular to the plane of the MXene slab, was set to $16 \AA$, thus ensuring at least $10 \AA$ of vacuum width between the periodic copies of the cell, even after adsorbing a small molecule. For $\mathrm{M}_{4} \mathrm{X}_{3}$ MXenes, a height of $20 \AA$ was used, so as to ensure the commented minimum vacuum region.

In order to study the adsorption of $\mathrm{N}_{2}$ (or $\mathrm{N}$ ) on the MXene surfaces, in a first approach, the adsorbate was placed far from the surface and allowed to adsorb onto it as per the attractive forces between adsorbent and adsorbate. This provided us with an initial guess of the most stable adsorbed configuration. Then, several other orientations and positions relatively to the surface were considered and their atomic positions fully relaxed. The 18 ground state geometries of $\mathrm{N}_{2}$ (or $\mathrm{N}$ ) adsorbed on each MXene were then established by comparing the total energy of the local minima configurations or, equivalently, by picking the one with the most negative adsorption energy. For each species $\mathrm{N}_{x}(x=1$ or 2$)$, the adsorption energy $\left(E_{\text {ads }}\right)$ is defined as

$$
E_{\mathrm{ads}}=E_{\mathrm{N}_{x} / \mathrm{MXene}}-\left(E_{\mathrm{MXene}}+E_{\mathrm{N}_{x}}\right)
$$

where $E_{\mathrm{N}_{x} / \mathrm{MXene}}, E_{\mathrm{MXene}}$, and $E_{\mathrm{N}_{x}}$ are the total energy of the MXene containing the adsorbed species, the bare pristine MXene, and the isolated $\mathrm{N}_{x}$ species, respectively. The energy of the isolated nitrogen atom was taken as $E_{\mathrm{N}}=\frac{1}{2} E_{\mathrm{N}_{2}}$.

The local minimum character of the ground state configurations was further verified by calculating the normal modes of vibration of the adsorbed species and ensuring that all the eigenvalues of the Hessian matrix were positive, and therefore correspond to real frequencies. The vibrational frequencies were obtained by diagonalization of the corresponding block of the Hessian matrix with elements computed as finite differences of $0.015 \AA$ of analytical gradients. The calculated frequencies were also used to calculate the zero-point energy (ZPE) contribution to the total energy of both adsorbed and gas phase species. The 
ZPE contributions changed the adsorption energies by less than $0.05 \mathrm{eV}$ and reduced the dissociation energy barriers by up to $0.06 \mathrm{eV}$.

The saddle point configuration for the minimum-energy pathway for $\mathrm{N}_{2}$ dissociation on each MXene, schematically shown in Figure 2, was located by using the dimer method. ${ }^{3}$ The transition state (TS) structure characterized by the frequency analysis ensured a single imaginary frequency corresponding to the N-N bond scission. This was verified by bidirectionally following the eigenvector of the dynamic matrix corresponding to the imaginary frequency, which yielded the two minima adjacent to the saddle point - a molecularly adsorbed $\mathrm{N}_{2}$, and two co-adsorbed $\mathrm{N}$ adatoms. The dissociation energy barrier, $E_{\mathrm{b}}$, is defined as the energy difference between the TS and the adsorbed $\mathrm{N}_{2}$ state, both including the ZPE term. The dissociation reaction energy was calculated as the energy difference between the $2 \mathrm{~N}$ adatoms co-adsorbed and the $\mathrm{N}_{2}$ adsorbed situations. Figure $\mathrm{S} 1$ shows the transition states of the formation of $\mathrm{NH}, \mathrm{NH}_{2}$ and $\mathrm{NH}_{3}$ and the respective most stable adsorbed configurations of these species, on $\mathrm{W}_{2} \mathrm{~N}$.

In order to include the effect of temperature and $\mathrm{N}_{2}$ partial pressure into the analysis, we calculated the variation of Gibbs free energy of the system upon adsorption of $\mathrm{N}_{2}\left(G_{\mathrm{ads}}\right)$, the adsorbed $\mathrm{N}_{2}$ dissociation transition state $\left(G_{\mathrm{TS}}\right)$, and the dissociated situation with $2 \mathrm{~N}$ adatoms $\left(G_{2 \mathrm{~N}}\right)$, considering as well the $G_{\text {ads }}$ of a single $\mathrm{N}$ atom. The Gibbs energy of adsorption of a $\mathrm{N}_{x}$ species was then calculated as

$$
G_{\mathrm{ads}}=E_{\mathrm{ads}}-k_{\mathrm{B}} T \ln \frac{q_{\mathrm{ads}}}{q_{\mathrm{gas}}}
$$

where $k_{\mathrm{B}}$ and $T$ are the Boltzmann constant and the absolute temperature, respectively. The quantity $q_{\text {ads }}\left(q_{\text {gas }}\right)$ is the partition function of the $\mathrm{N}_{x}$ species in its adsorbed (gas phase) configuration. For the isolated $\mathrm{N}$, we assumed $q_{\text {gas }}(N)=\sqrt{q_{\text {gas }}\left(N_{2}\right)}$, so that the resulting $G_{\text {ads }}$ has the same value as when one considers the co-adsorption of two $\mathrm{N}$ atoms and divides the result by two. For the gas phase molecule, the partition function includes vibrational, rotational, and translational contributions $\left(q=q_{\mathrm{vib}} \cdot q_{\mathrm{rot}} \cdot q_{\mathrm{trans}}\right)$, while in the adsorbed phase only the vibrational contribution is considered, given that the molecular translations and rotations become frustrated upon adsorption. The vibrational partition function, choosing the bottom of the internuclear potential energy well as the zero-reference point, i.e. non ZPE corrected, can be calculated knowing the frequencies $v_{i}$ of the normal vibrational modes,

$$
q_{\mathrm{vib}}=\prod_{i} \frac{\exp \left(-h v_{i} /\left(2 k_{B} T\right)\right)}{1-\exp \left(-h v_{i} /\left(k_{B} T\right)\right)}
$$

where $h$ is the Planck constant. For a linear molecule, such as $\mathrm{N}_{2}$, the rotational and translational contributions to the partition function are given by

$$
q_{\mathrm{rot}} \approx \frac{1}{\sigma} \frac{T}{\theta_{\mathrm{rot}}}
$$


and, finally, the translational partition function is

$$
q_{\text {trans }}=\left(\frac{2 \pi m k_{B} T}{h^{2}}\right)^{3 / 2} \frac{k_{B} T}{p_{N_{2}}}
$$

respectively. Here, $\sigma=2$ is the symmetry number for $\mathrm{N}_{2}$ that is common to all linear molecules with a center of inversion, with symmetry group $C_{\infty h}$, while $\theta_{\text {rot }}=2.78 \mathrm{~K}$ is the rotational temperature of $\mathrm{N}_{2}$, obtained by calculating the principal moment of inertia of the linear molecule in its optimized geometry, $m$ is the mass of the molecule, and $p_{\mathrm{N}_{2}}$ is the $\mathrm{N}_{2}$ partial pressure. In this work, the temperature and $\mathrm{N}_{2}$ partial pressure were fixed at $300 \mathrm{~K}$ and 1 bar, respectively.

The Gibbs free energy for the dissociation transition state relative to the adsorption ground state, $G_{\mathrm{b}}$, is calculated like $G_{\mathrm{ads}}$, but replacing the adsorption energy with the energy barrier, and the quotient of partition functions by $q_{\mathrm{TS}} / q_{\mathrm{ads}}$, including only the vibrational terms. Finally, the Gibbs free energy of dissociation, $G_{\text {reac, }}$ is calculated like $E_{\text {reac, }}$ using Gibbs free energies of adsorption.

\section{S2. Additional comparison to previous work}

Qualitatively, the adsorption behavior of $\mathrm{N}_{2}$ on $\mathrm{Ti}$, Ta, Mo, and $\mathrm{W}$ carbides and nitrides coincides with the previous findings by Shao et al. ${ }^{4} \mathrm{We}$ also observed the adsorption-induced slight distortion of the $\mathrm{Mo}_{2} \mathrm{~N}$ and $\mathrm{W}_{2} \mathrm{~N}$ lattices. Curiously, we obtained the same kind of distortion on $\mathrm{W}_{2} \mathrm{C}$ and $\mathrm{Cr}_{2} \mathrm{~N}$. However, the distortion was considered too small to justify the exclusion of these MXenes from the analysis, especially because all surfaces relaxed back to their original configurations upon removal of $\mathrm{N}_{2}$. Quantitatively, and also in agreement with Ref. 5, the absolute values of the binding energies of $\mathrm{N}_{2}$ to Ti- and Ta-based MXenes are about twice as high as on Mo- and W-based ones. Our calculated adsorption energy of $\mathrm{N}_{2}$ on $\mathrm{Ti}_{2} \mathrm{C}$ of -3.26 $\mathrm{eV}$ agrees with the $-3.52 \mathrm{eV}$ found by $\mathrm{Yi}$ et al. on the adsorption and dissociation behavior of $\mathrm{N}_{2}$ on $\mathrm{Ti}_{2} \mathrm{C}^{5}$ In that work, the nitrogen bond length is predicted to increase from 1.11 to $1.35 \AA$ upon adsorption, also in agreement with our corresponding calculations, 1.11 and $1.36 \AA$, respectively.

\section{S3. Ammonia synthesis}

Ammonia synthesis is analyzed following the scheme reported by Honkala et al., ${ }^{6}$ considering the global reaction $\mathrm{N}_{2}+3 \mathrm{H}_{2} \rightarrow 2 \cdot \mathrm{NH}_{3}$. The energy barriers of the $\mathrm{N}_{2}$ dissociation and the subsequent hydrogenation steps to form $\mathrm{NH}_{3}$ are compiled in Table $\mathrm{S} 3$, whereas the total and Gibbs free energy reaction profiles are shown in Figure 4. MXenes show lower energy barriers for $\mathrm{N}_{2}$ dissociation than the $\mathrm{Ru}(001)$ surface. However, the hydrogenation energy barriers on the Ru (001) surface are lower than on MXenes.

\section{S4. Microkinetic simulations}

The calculations of the forward and backward rate constants of the elementary steps were performed by using the following equation, 


$$
k=\frac{k_{B} T}{h} \frac{Q^{T S}}{Q} e^{-\frac{E_{a}}{k_{B} T}}
$$

The partition function $(Q)$ corresponds to the summation of all possible states including translation, rotation, and vibration modes. The vibrational degree of freedom becomes dominating for surface reactions, and the pre-exponential factor is governed by the $k_{\mathrm{B}} T / h$. The flux of the incident molecule is given by the Hertz-Knudsen equation ${ }^{7}$

$$
F=\frac{P}{\sqrt{2 \pi m k_{B} T}}
$$

and the molecular adsorption rate constant is expressed as

$$
k_{a d s}=-\frac{P A^{\prime}}{\sqrt{2 \pi m k_{B} T}} S
$$

where $P$ is the partial pressure of the molecule, $S$ is the sticking coefficient, which is assumed to be $1, A^{\prime}$ is the area of an adsorption site $-6.7 \times 10^{-20} \mathrm{~m}^{2}$ on the $\mathrm{W}_{2} \mathrm{~N}$ surface- , and $m$ is the mass of the molecule. On the other hand, three rotational degrees and two translational degrees of freedom are assumed in the case of the desorption in the transition state.

$$
k_{d e s}=\frac{k_{B} T^{3}}{h^{3}} \frac{A^{\prime}\left(2 \pi k_{B}\right)}{\sigma \theta_{\text {rot }}} e^{-\frac{E_{d e s}}{k_{B} T}}
$$

Microkinetic simulations were performed with the MKMCXX program, ${ }^{8,9}$ employing an initial gaseous mixture of $\mathrm{N}_{2}$ and $\mathrm{H}_{2}$ in a 1:3 molar ratio at a total pressure of 1 or 200 bar. 
Table S1. Calculated lattice parameters, $a$, in $\AA$, of the 18 MXenes studied.

\begin{tabular}{c|cc}
\hline $\mathbf{M}$ & $\mathbf{C}$ & $\mathbf{N}$ \\
\hline \hline $\mathbf{T i}$ & 3.06 & 2.98 \\
$\mathbf{V}$ & 2.90 & 2.89 \\
$\mathbf{C r}$ & 2.82 & 2.68 \\
$\mathbf{Z r}$ & 3.27 & 3.24 \\
$\mathbf{N b}$ & 3.13 & 3.15 \\
$\mathbf{M o}$ & 3.10 & 2.79 \\
$\mathbf{H f}$ & 3.21 & 3.17 \\
$\mathbf{T a}$ & 3.09 & 3.09 \\
$\mathbf{W}$ & 2.88 & 2.78 \\
\hline
\end{tabular}


Table S2. Adsorption energy at the most stable site for atomic N on the MXene (0001) surfaces. The labelling for adsorption sites is shown in Figure 1. The reference energy for $\mathrm{N}$ in gaseous phase has been chosen as $E_{\mathrm{N}}=\frac{1}{2} E_{\mathrm{N}_{2}}$. All values are given in $\mathrm{eV}$ and include the ZPE contribution.

\begin{tabular}{c|ccccc}
\hline \multicolumn{1}{c}{} & M & \multicolumn{2}{c}{$\mathbf{M}_{2} \mathbf{C}$} & \multicolumn{2}{c}{$\mathbf{M}_{2} \mathbf{N}$} \\
\hline \hline \multirow{4}{*}{$\boldsymbol{d}^{\mathbf{2}}$} & $\mathrm{Ti}$ & $\mathrm{H}_{\mathrm{M}}$ & -2.40 & $\mathrm{H}_{\mathrm{M}}$ & -2.72 \\
& $\mathrm{Zr}$ & $\mathrm{H}_{\mathrm{M}}$ & -2.22 & $\mathrm{H}_{\mathrm{M}}$ & -2.67 \\
& $\mathrm{Hf}$ & $\mathrm{H}_{\mathrm{M}}$ & -2.58 & $\mathrm{H}_{\mathrm{M}}$ & -2.95 \\
\hline \multirow{4}{*}{$\boldsymbol{d}^{\mathbf{3}}$} & $\mathrm{V}$ & $\mathrm{H}_{\mathrm{M}}$ & -2.38 & $\mathrm{H}_{\mathrm{M}}$ & -2.15 \\
& $\mathrm{Nb}$ & $\mathrm{H}_{\mathrm{M}}$ & -2.30 & $\mathrm{H}_{\mathrm{M}}$ & -2.23 \\
& $\mathrm{Ta}$ & $\mathrm{H}_{\mathrm{M}}$ & -2.54 & $\mathrm{H}_{\mathrm{M}}$ & -2.59 \\
\hline \multirow{4}{*}{$\boldsymbol{d}^{\mathbf{4}}$} & $\mathrm{Cr}$ & $\mathrm{H}_{\mathrm{M}}$ & -2.01 & $\mathrm{H}_{\mathrm{N}}$ & -1.76 \\
& $\mathrm{Mo}$ & $\mathrm{H}_{\mathrm{M}}$ & -1.97 & $\mathrm{H}_{\mathrm{N}}$ & -2.15 \\
& $\mathrm{~W}$ & $\mathrm{H}_{\mathrm{C}}$ & -1.79 & $\mathrm{H}_{\mathrm{N}}$ & -2.26 \\
\hline
\end{tabular}


Table S3. Forward energy barriers, $E_{\mathrm{b}}$, in $\mathrm{eV}$, of the total energy reaction profile, see Figure 4, corresponding to the dissociation of $\mathrm{N}_{2}$ molecule and to the subsequent hydrogenation reactions required to form ammonia over $\mathrm{Nb}_{2} \mathrm{C}, \mathrm{Mo}_{2} \mathrm{~N}$, and $\mathrm{W}_{2} \mathrm{~N}$. The results reported by Logadóttir and Nørskov. ${ }^{10}$ for the same reaction steps on two different surface regions of the $\mathrm{Ru}(0001)$ catalyst model are included for comparison.

\begin{tabular}{|c|c|c|c|c|}
\hline & $\begin{array}{c}\mathrm{N}_{2} \\
\text { dissociation }\end{array}$ & $\begin{array}{c}\text { 1 }^{\text {st }} \text { hydrogenation } \\
\text { NH }\end{array}$ & $\begin{array}{c}2^{\text {nd }} \text { hydrogenation } \\
\qquad \mathrm{NH}_{2}\end{array}$ & $\begin{array}{c}3^{\text {rd }} \text { hydrogenation } \\
\mathrm{NH}_{3}\end{array}$ \\
\hline \multicolumn{5}{|l|}{ MXenes } \\
\hline $\mathrm{Nb}_{2} \mathrm{C}$ & 0.75 & 1.73 & 1.82 & 2.56 \\
\hline $\mathrm{Mo}_{2} \mathrm{~N}$ & 0.45 & 1.35 & 1.79 & 2.12 \\
\hline $\mathrm{W}_{2} \mathrm{~N}$ & 0.28 & 1.24 & 1.70 & 1.69 \\
\hline \multicolumn{5}{|l|}{ Ru (0001) } \\
\hline Step & 0.40 & 1.10 & 1.30 & 1.20 \\
\hline Terrace & 1.99 & 1.20 & 1.30 & 1.40 \\
\hline
\end{tabular}


Table S4. Energy barriers, $E_{\mathrm{b}}$, in $\mathrm{eV}$, of the Gibbs free energy reaction profile, see Figure 4 , assuming a temperature of $300 \mathrm{~K}$ and $\mathrm{N}_{2}$ and $\mathrm{H}_{2}$ partial pressures of 1 bar.

\begin{tabular}{|c|c|c|c|c|}
\hline & $\begin{array}{c}\mathrm{N}_{2} \\
\text { dissociation }\end{array}$ & $\begin{array}{c}1^{\text {st }} \text { hydrogenation } \\
\text { NH }\end{array}$ & $\begin{array}{c}2^{\text {nd }} \text { hydrogenation } \\
\mathrm{NH}_{2}\end{array}$ & $\begin{array}{c}3^{\text {rd }} \text { hydrogenation } \\
\mathrm{NH}_{3}\end{array}$ \\
\hline \multicolumn{5}{|c|}{ MXenes } \\
\hline $\mathrm{Nb}_{2} \mathrm{C}$ & 0.65 & 1.67 & 1.74 & 2.47 \\
\hline $\mathrm{Mo}_{2} \mathrm{~N}$ & 0.43 & 1.31 & 1.73 & 2.06 \\
\hline $\mathrm{W}_{2} \mathrm{~N}$ & 0.23 & 1.17 & 1.60 & 1.62 \\
\hline
\end{tabular}


Table S5. Comparison of the activation energy barriers (in eV) corresponding to $\mathrm{Ru}$ based catalyst ${ }^{11}$ and $\mathrm{W}_{2} \mathrm{~N}$ MXene (0001) surface for the forward and backward processes involved in the $\mathrm{N}_{2}$ fixation. The backward reaction to form $\mathrm{N}_{2}$ has two barriers: 1.16 and $3.46 \mathrm{eV}$ that correspond to the situations of the $\mathrm{N}^{*}$ species located nearby or far from each other, respectively.

\begin{tabular}{lcc}
\hline & \multicolumn{2}{c}{$\mathrm{Ru} / \mathbf{W}_{2} \mathbf{N}$} \\
\cline { 2 - 3 } $\mathrm{N}_{2} \rightarrow 2 \mathrm{~N}^{*}$ & Forward & Backward \\
\cline { 2 - 3 } $\mathrm{H}_{2} \rightarrow 2 \mathrm{H}^{*}$ & $0.34 / \mathbf{0 . 2 8}$ & $1.42 / \mathbf{1 . 1 2}(\mathbf{3 . 4 6})$ \\
$\mathrm{N}^{*}+\mathrm{H}^{*} \rightarrow \mathrm{NH}^{*}$ & $0 / \mathbf{0 . 0 4}$ & $0.92 / \mathbf{0 . 8 6}$ \\
$\mathrm{NH}^{*}+\mathrm{H}^{*} \rightarrow \mathrm{NH}_{2}^{*}$ & $0.89 / \mathbf{1 . 2 4}$ & $0.42 / \mathbf{0 . 8 1}$ \\
$\mathrm{NH}_{2} *+\mathrm{H}^{*} \rightarrow \mathrm{NH}_{3} *$ & $0.17 / \mathbf{1 . 6 9}$ & $0.09 / \mathbf{1 . 0 2}$ \\
$\mathrm{NH}_{3}^{*} \rightarrow \mathrm{NH}_{3}$ & $0.87 / \mathbf{1 . 5 3}$ & $0.67 / \mathbf{0 . 4 7}$ \\
\hline
\end{tabular}


Figure S1. Transition state (left half of the page) and ground state adsorption (right half) configurations of $\mathrm{NH}$ (top), $\mathrm{NH}_{2}$ (middle), and $\mathrm{NH}_{3}$ (bottom) during the process of triple hydrogenation of a nitrogen adatom on $\mathrm{W}_{2} \mathrm{~N}$. Spheres represent atoms of hydrogen (white), adsorbed nitrogen (navy), MXene inner nitrogen (green), and tungsten (grey).
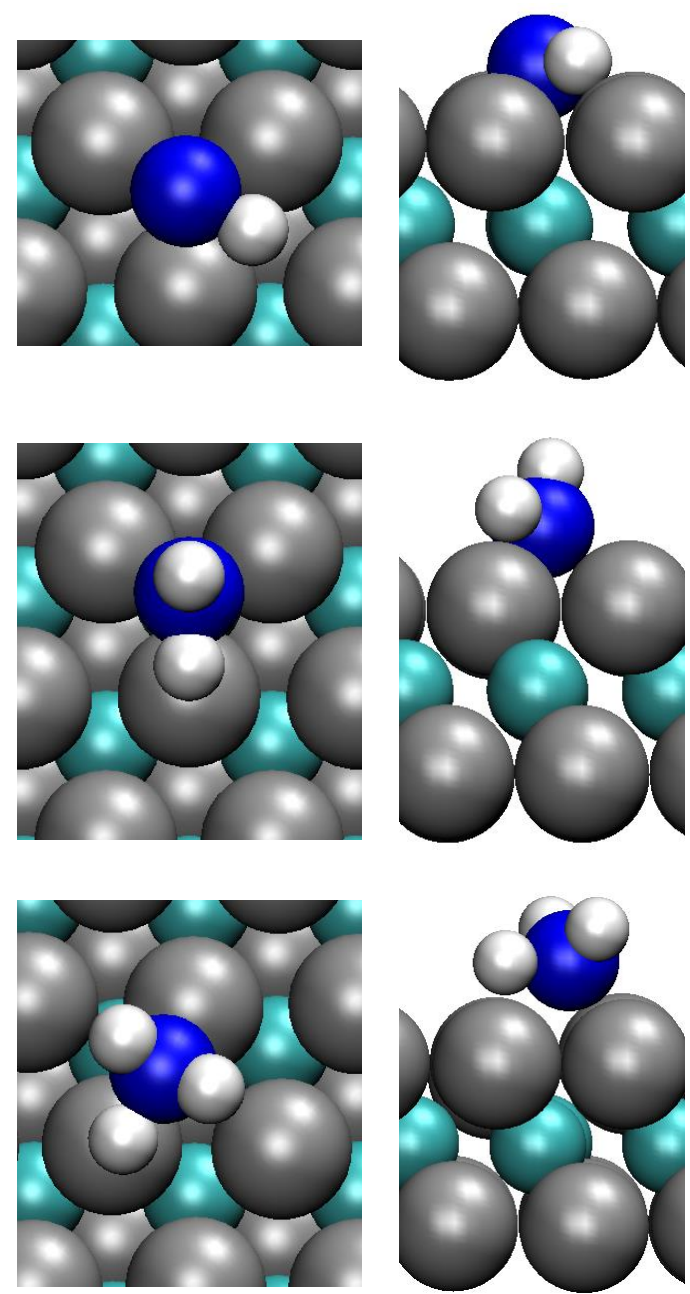
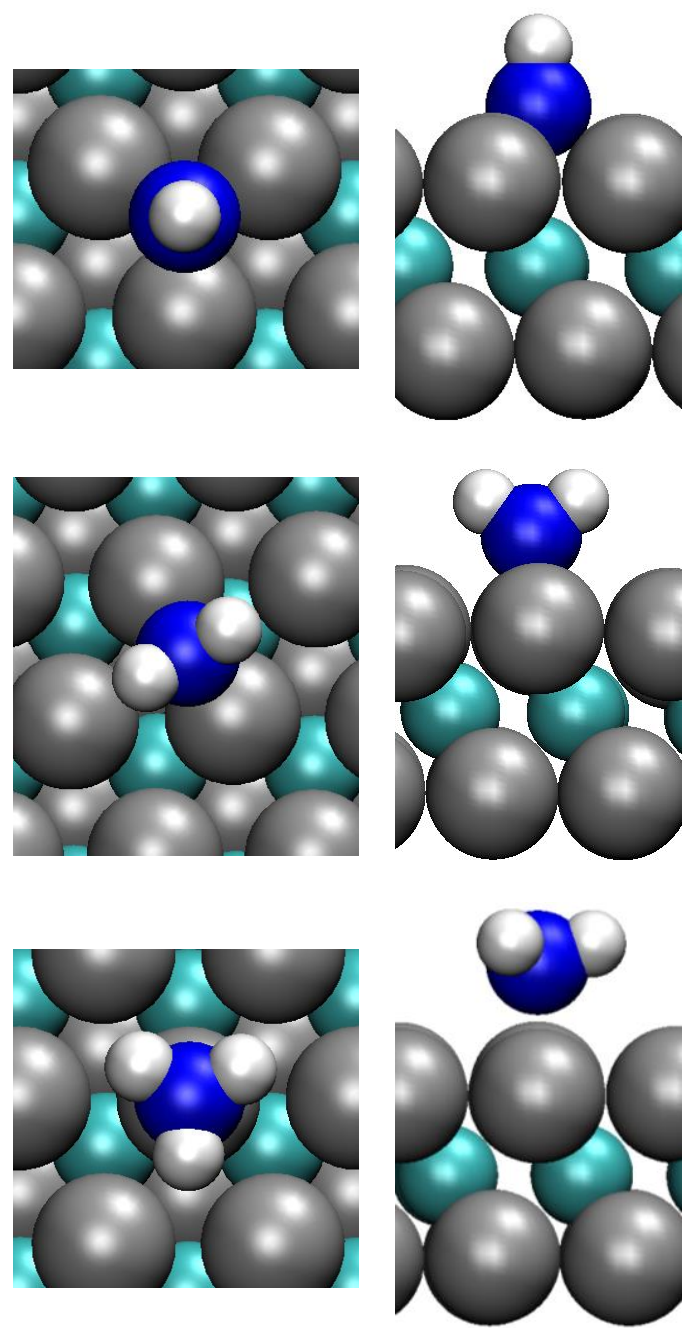
Figure S2. Apparent activation energy (in $J / m o l$ ) of the $\mathrm{N}_{2}$ fixation over the $\mathrm{W}_{2} \mathrm{~N}$ MXene (0001) surface resulting from the microkinetic simulations for the situation where the $\mathrm{N}^{*}$ species are located nearby. The initial gas phase contains a mixture of $\mathrm{N}_{2}$ and $\mathrm{H}_{2}$ in a 1:3 molar ratio at a total pressure of 1 bar.

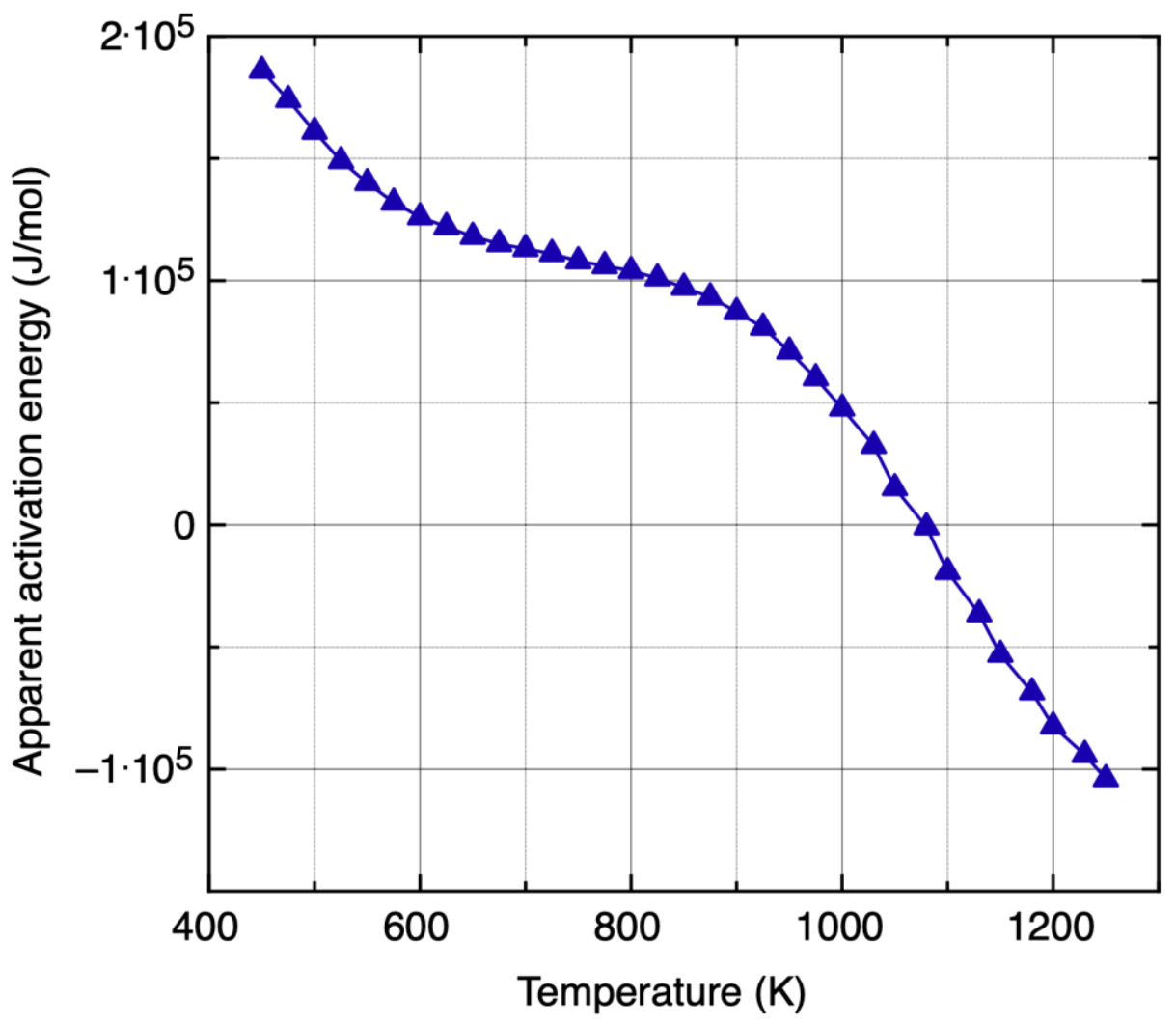


Figure S3. Reaction order of the $\mathrm{N}_{2}$ fixation over the $\mathrm{W}_{2} \mathrm{~N}$ MXene (0001) surface resulting from the microkinetic simulations for the situation where the $\mathrm{N}^{*}$ species are located nearby. The initial gas phase contains a mixture of $\mathrm{N}_{2}$ and $\mathrm{H}_{2}$ in a 1:3 molar ratio at a total pressure of 1 bar.

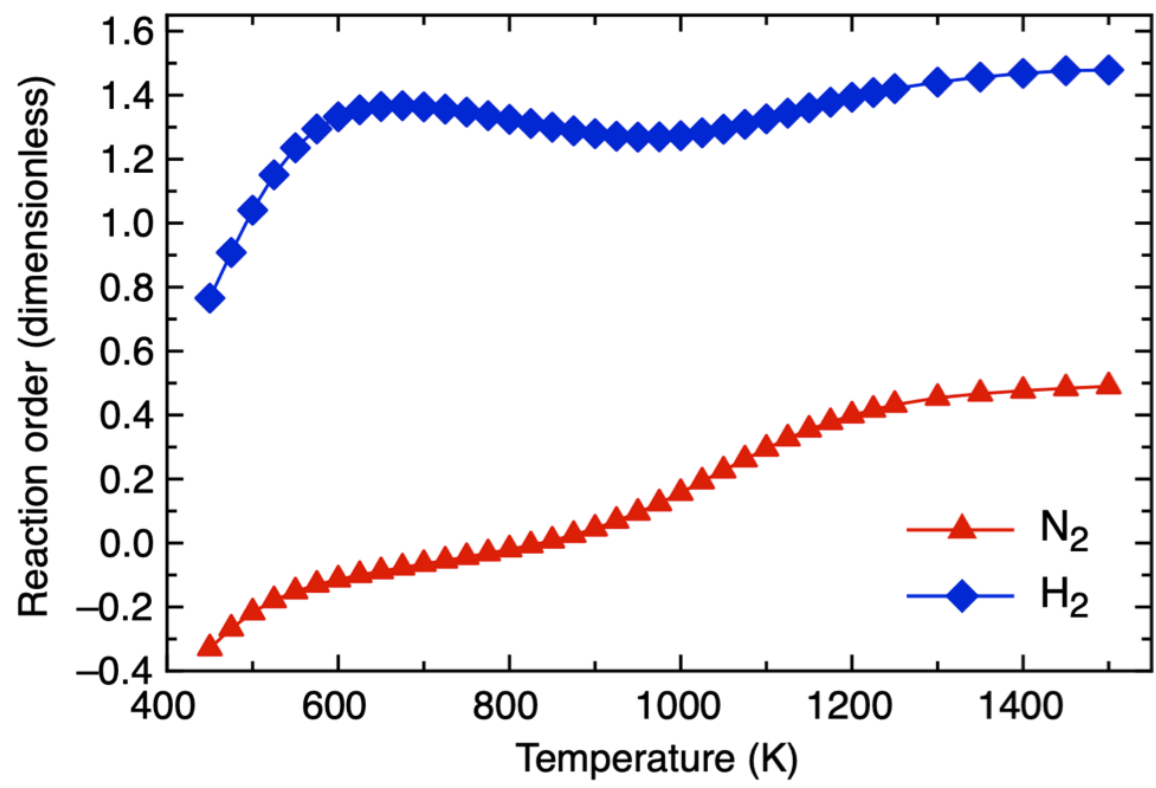


Figure S4. DRC coefficient of the $\mathrm{N}_{2}$ fixation over the $\mathrm{W}_{2} \mathrm{~N}$ MXene (0001) surface resulting from the microkinetic simulations for the situation where the $\mathrm{N}^{*}$ species are located nearby. The initial gas phase contains a mixture of $\mathrm{N}_{2}$ and $\mathrm{H}_{2}$ in a 1:3 molar ratio at a total pressure of 1 bar.

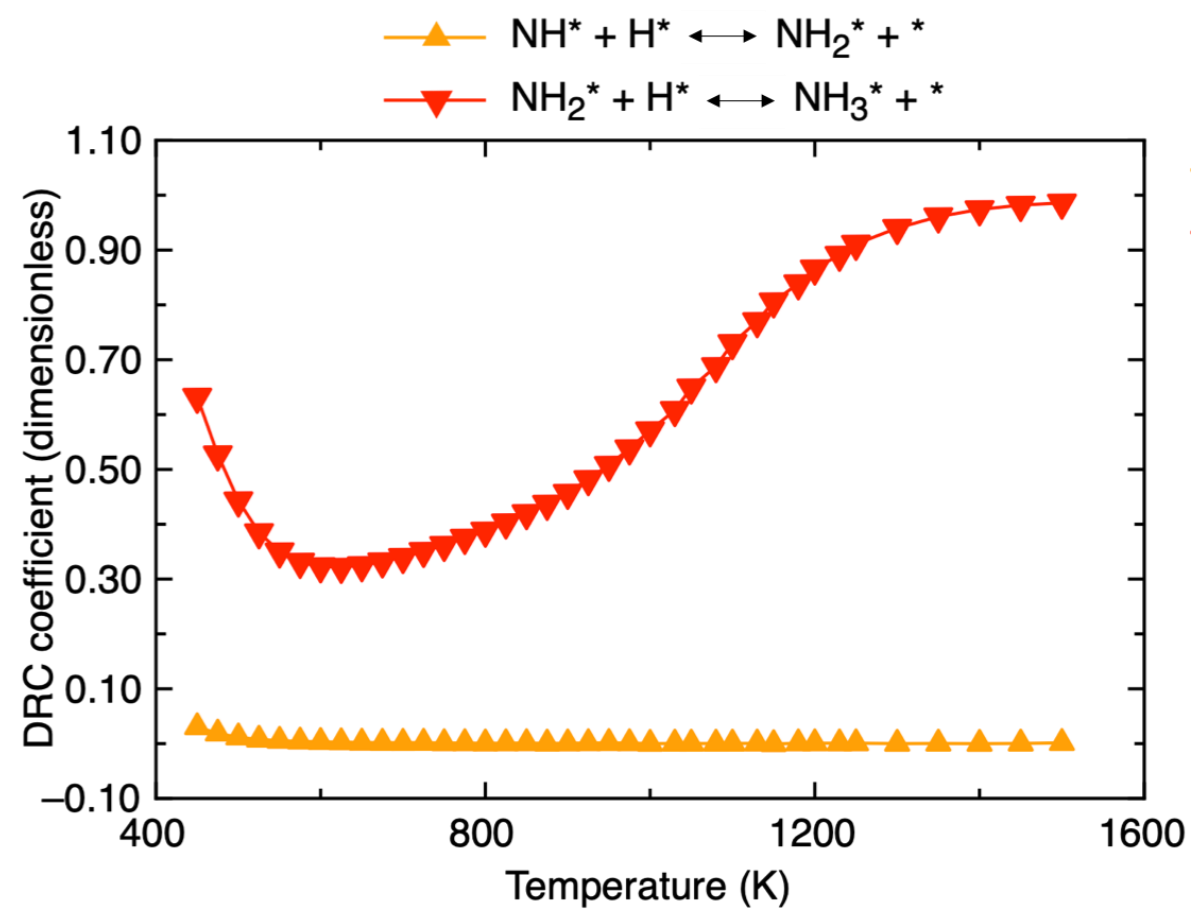


Figure S5. Rates of forward and backward reactions involved in the $\mathrm{N}_{2}$ fixation over the $\mathrm{W}_{2} \mathrm{~N}$ MXene (0001) surface resulting from the microkinetic simulations for the situation where the $\mathrm{N}^{*}$ species are located nearby. The initial gas phase contains a mixture of $\mathrm{N}_{2}$ and $\mathrm{H}_{2}$ in a 1:3 molar ratio at a total pressure of 1 bar. Note that (b) corresponds to a zoom-in of (a).
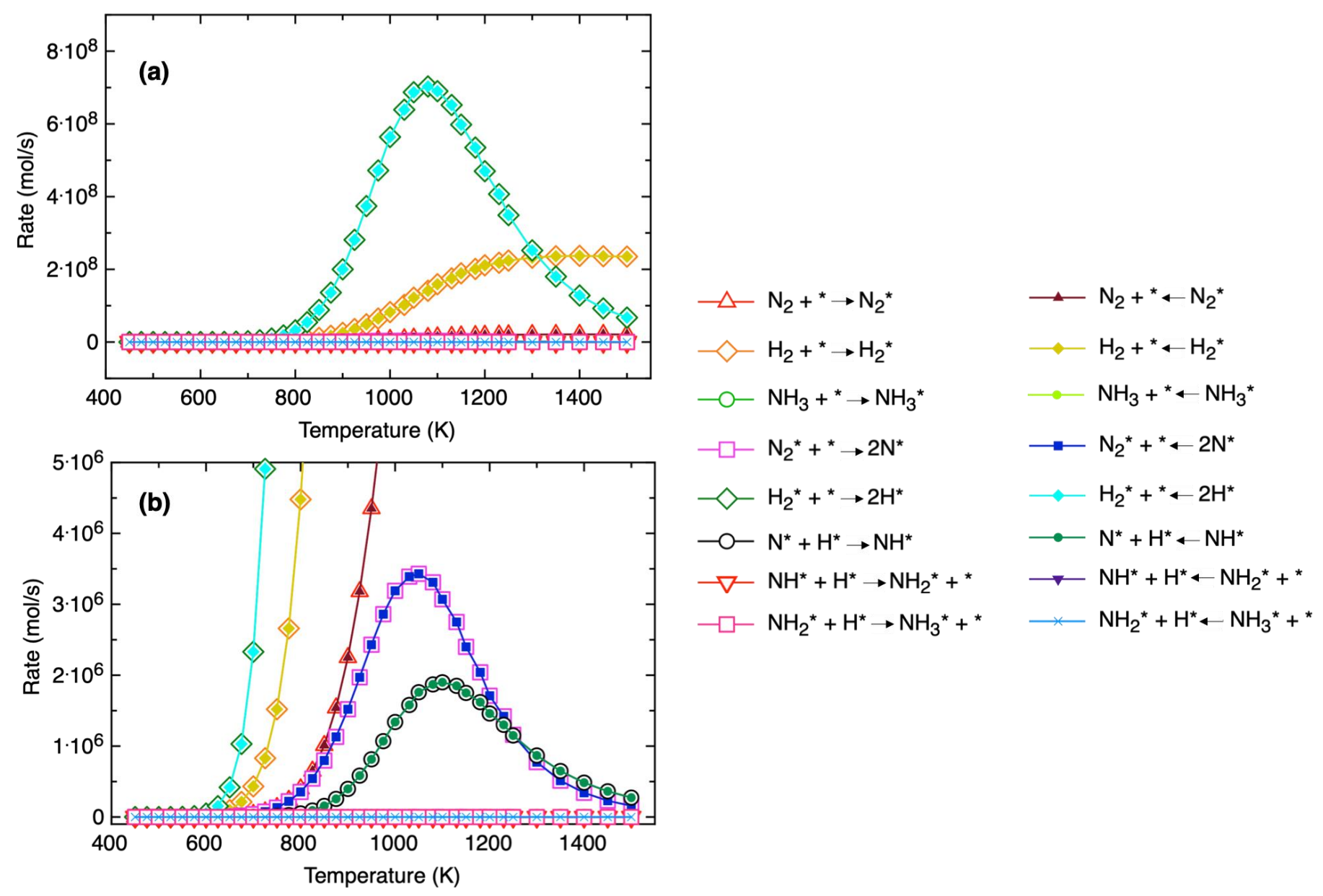
Figure S6. Results from microkinetic simulations performed on the $\mathrm{W}_{2} \mathrm{~N}$ MXene (0001) for the scenario where the $\mathrm{N}^{*}$ species are close to each other (high-coverage). The initial gas phase contains a mixture of $\mathrm{N}_{2}$ and $\mathrm{H}_{2}$ in a 1:3 molar ratio at a total pressure of 200 bar. The coverage of the most abundant species for the $\mathrm{N}_{2}$ fixation on the MXene surface is shown in the left panel, while the ammonia production as a function of the temperature is shown in the right panel.
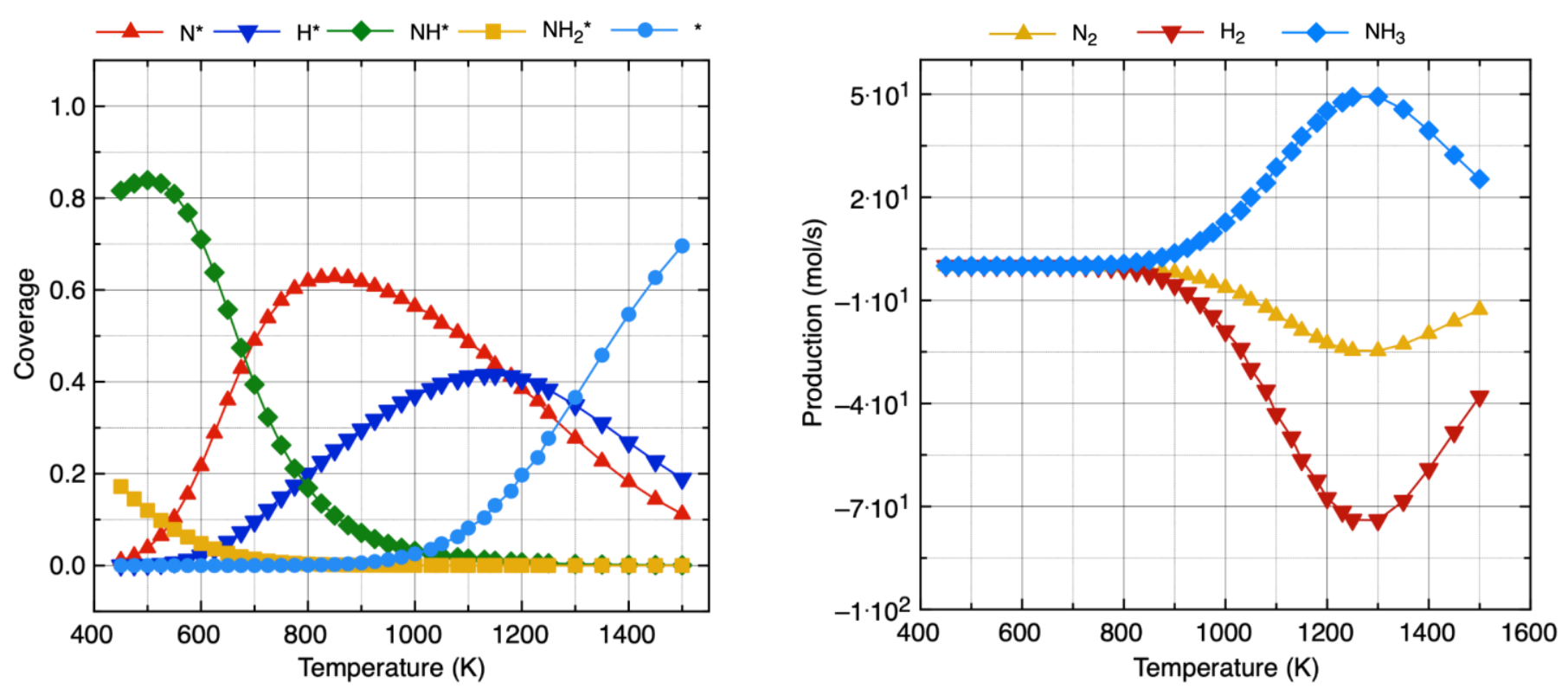
Figure S7. Apparent activation energy (in $\mathrm{J} / \mathrm{mol}$ ) of the $\mathrm{N}_{2}$ fixation over the $\mathrm{W}_{2} \mathrm{~N}$ MXene (0001) surface resulting from the microkinetic simulations for the situation where the $\mathrm{N}^{*}$ species are located nearby. The initial gas phase contains a mixture of $\mathrm{N}_{2}$ and $\mathrm{H}_{2}$ in a 1:3 molar ratio at a total pressure of 200 bar.

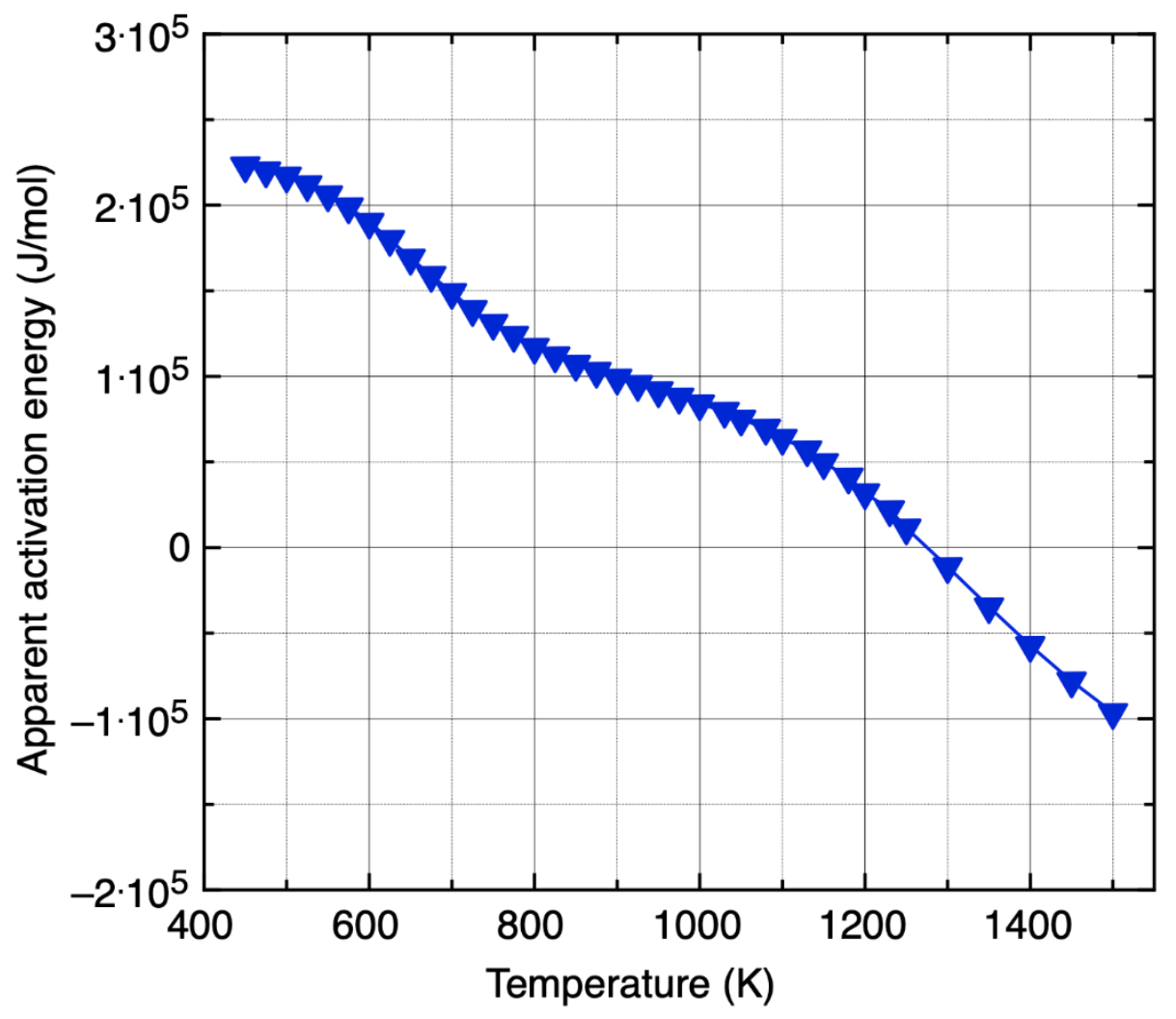


Figure S8. Reaction order of the $\mathrm{N}_{2}$ fixation over the $\mathrm{W}_{2} \mathrm{~N}$ MXene (0001) surface resulting from the microkinetic simulations for the situation where the $\mathrm{N}^{*}$ species are located nearby. The initial gas phase contains a mixture of $\mathrm{N}_{2}$ and $\mathrm{H}_{2}$ in a 1:3 molar ratio at a total pressure of 200 bar.

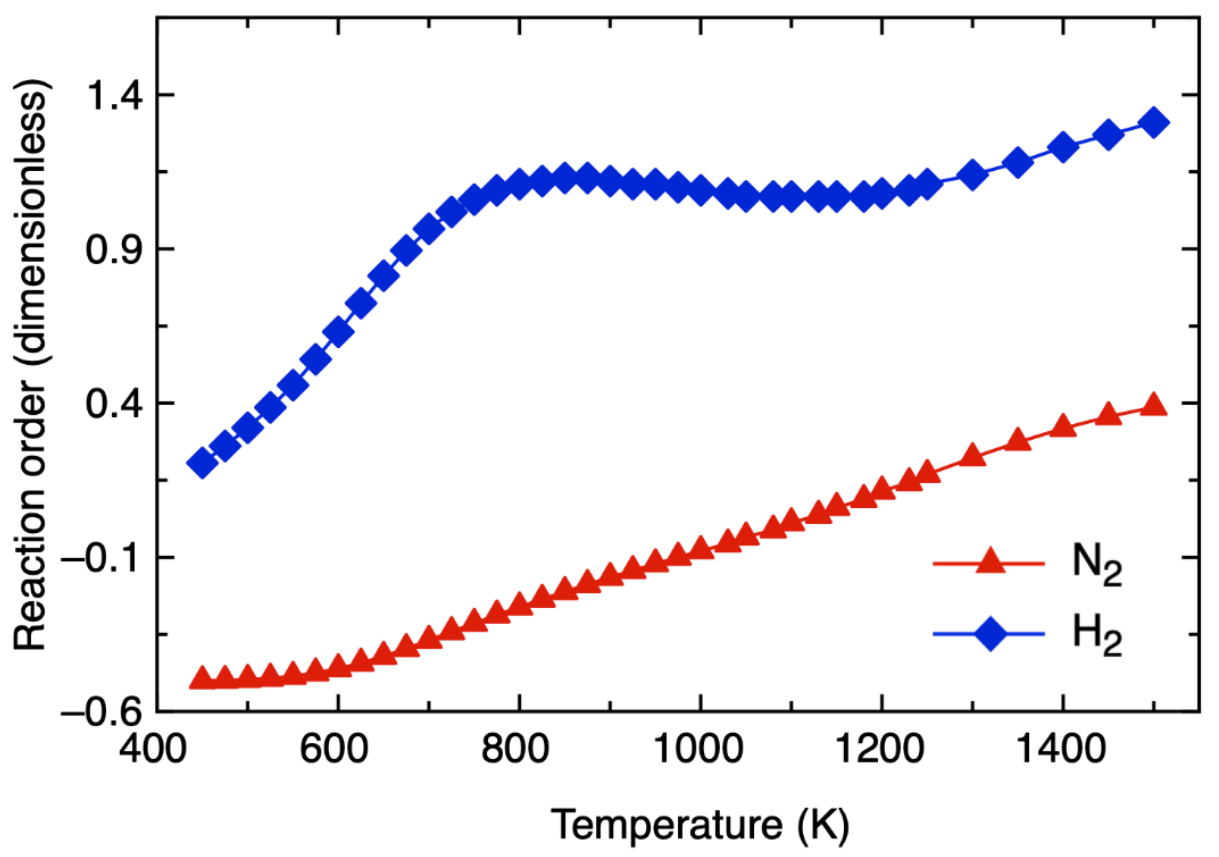




\section{References}

(1) Zhang, N.; Hong, Y.; Yazdanparast, S.; Zaeem, M. A. Superior Structural, Elastic and Electronic Porperties of 2D Titanium Nitride MXenes over Carbide MXenes: A Comprenhensive First Principles Study. 2D Mater. 2018, 5, 045004.

(2) Naguib, M.; Mochalin, V. N.; Barsoum, M. W.; Gogotsi, Y. 25 ${ }^{\text {th }}$ Anniversary Article: MXenes:A New Family of Two-Dimensional Materials. Adv. Mater. 2014, 26, 992-1005.

(3) Henkelman, G.; Jónsson, H. A Dimer Method for Finding Saddle Points on High Dimensional Potential Surfaces Using Only First Derivatives. J. Chem. Phys. 1999, 111, 7010-7022.

(4) Shao, M.; Shao, Y.; Chen, W.; Ao, K. L.; Tong, R.; Zhu, Q.; Chan, I. N.; Ip, W. F.; Shi, X.; Pan, H. Efficient Nitrogen Fixation to Ammonia on MXenes. Phys. Chem. Chem. Phys. 2018, 20, 14504-14512.

(5) Yi, S.; Liu, G.; Liu, Z.; Hu, W.; Deng, H. Theoretical Insights into Nitrogen Fixation on $\mathrm{Ti}_{2} \mathrm{C}$ and $\mathrm{Ti}_{2} \mathrm{CO}_{2}$ in a Lithium-Nitrogen Battery. J. Mater. Chem. A 2019, 7, 19950-19960.

(6) Honkala, K.; Hellman, A.; Remediakis, I. N.; Logadóttir, A; Carlsson, A.; Dahl, S.; Christensen, C. H.; Nørskov, J. K. Ammonia Synthesis from First-Principles Calculations. Science 2005, 307, 555-558.

(7) Niton, P.; Zywocinski, A.; Fialkowski, M.; Holyst, R. A "Nanowindmill” Driven by a Flux of Water Vapour: A Comparison to the Rotating ATPase. Nanoscale 2013, 5, 9732-9738.

(8) Filot, I. A. W.; van Santen, R. A.; Hensen, E. J. M. The Optimally Performing Fischer-Tropsch Catalyst. Angew. Chem., Int. Ed. 2014, 53, 12746-12750.

(9) Filot, I. A. W.; Broos, R. J. P.; van Rijn, J. P. M.; van Heugten, G. J. H.; van Santen, R. A.; Hensen, E. J. M. FirstPrinciples-Based Microkinetics Simulations of Synthesis Gas Conversion on a Stepped Rhodium Surface. ACS Catal. 2015, 5, 5453-5467.

(10) Logadóttir, Á.; Nørskov, J. Ammonia Synthesis Over a Ru(0001) Surface Studied by Density Functional Calculations. J. Catal. 2003, 220, 273-279.

(11) Hinrichsen, O.; Rosowski, F.; Muhler, M.; Ertl, G. The Microkinetics of Ammonia Synthesis Catalyzed by Cesium-Promoted Supported Ruthenium. Chem. Eng. Sci. 1996, 51, 1683-1690. 